\title{
Action combinée de l'aclonifen et de la bentazone administrés avec ou sans formulation
}

\author{
F Cabanne *, JC Gaudry \\ INRA, laboratoire de phytopharmacie, BV 1540, F 21034 Dijon cedex, France
}

(Reçu le 21 juillet 1995 ; accepté le 31 octobre 1995)

\begin{abstract}
Résumé - Dans le cadre d'essais biologiques conduits en conditions contrôlées, on a traité des plantes de gaillet avec de l'aclonifen et de la bentazone. Les traitements mettaient en œuvre les matières actives seules ou en mélange, dissoutes dans une solution hydro-acétonique ou présentes dans leur formulation commerciale (Challenge 600 et Basagran). On a fait appel à 2 méthodes pour évaluer l'efficacité des mélanges. L'une utilise un plan factoriel et un modèle qui calcule une additivité d'effets. L'autre procède à partir de courbes de croissance et calcule une additivité d'effets ou une additivité de doses. L'aclonifen et la bentazone administrés en mélange (rapport pondéral 1,25) produisent un antagonisme aux faibles doses et une synergie aux doses élevées. Les produits formulés (mélangés dans le même rapport) donnent également ces 2 types d'effets. Cependant, les produits formulés manifestent une synergie qui est plus prononcée que celle des matières actives et qui affecte les doses élevées produisant la destruction des plantes. On propose que l'efficacité des produits formulés additionne la synergie des matières actives et un effet croisé des formulants.
\end{abstract}

\section{herbicide / aclonifen / bentazone / synergie / gaillet}

Summary - Joint action of aclonifen and bentazone applied with or without formulation. Bioassays were carried out with cleavers plants treated with aclonifen and bentazone, in controlled conditions. The active ingredients were applied alone, as a mixture, dissolved in acetone/water, or as their commercial formulations (Challenge 600 and Basagran). The efficacy of mixtures was evaluated with 2 methods. One used a factorial design and calculated an additivity of effects. The other worked from response curves and was able to calculate an additivity of effects or an additivity of doses. When aclonifen and bentazone were mixed in a ratio of 1.25, an antagonism was detected at low doses and a synergism at high doses. The formulated products, mixed in the same ratio, gave the same types of effects. However, the synergism of the formulated products was stronger than that of the active ingredients and focused on the high doses inducing the death of plants. Thus, the efficacy of the formulated products would add the synergistic effect of active ingredients and a crossed effect of formulation agents.

herbicide / aclonifen / bentazone / synergism / cleavers

\footnotetext{
* Correspondance et tirés à part
} 


\section{INTRODUCTION}

Un traitement de post-semis pré-levée est la meilleure solution pour désherber le pois de printemps en France (Jouy et Leveau, 1992). Dans $40 \%$ des cas, il est constitué d'un mélange. II peut s'agir d'un mélange de matières actives dans une formulation homologuée ou d'un mélange d'herbicides réalisé par l'agriculteur au moment du traitement. Ces mélanges comprennent souvent une toluidine (trifluraline, pendiméthaline) et une urée (linuron, néburon, méthabenzthiazuron). Le mélange trifluraline + linuron permet de contrôler Alopecurus myosuroides, Chenopodium album, Matricaria recutita, Polygonum aviculare et Veronica sp. La pendiméthaline élargit le contrôle aux graminées estivales, le néburon à Lolium multiflorum et le méthabenzthiazuron à Aethusa cynapium. En cas d'infestation par Galium aparine, une espèce de très forte nuisibilité, l'aclonifen est conseillé à raison de $2700 \mathrm{~g} \mathrm{ha}^{-1}$ de matière active (ma).

Depuis quelques années, il est possible de désherber le pois avec un traitement de postlevée. Deux mélanges homologués sont disponibles sur le marché pour lutter contre les dicotylédones. L'un à base de diméfuron et de bentazone est recommandé contre $G$ aparine, $M$ recutita et Stellaria media (Jouy et Leveau, 1992). L'autre à base de pendiméthaline et de bentazone contrôle $M$ recutita et $S$ media et plus spécifiquement $P$ aviculare et Fumaria officinalis (Morvan et Petat, 1992).

Environ $20 \%$ des surfaces de pois désherbées en pré-levée nécessitent une intervention de rattrapage en post-levée (Jouy et Leveau, 1992). Par ailleurs, certaines cultures de pois faites dans le nord de la France présentent une infestation réduite malgré l'absence de traitement de pré-levée. Les mélanges commerciaux de postlevée, à base de diméfuron + bentazone ou de pendiméthaline + bentazone, n'apportent pas de réponse satisfaisante à ces cas de désherbage car ils présentent un coût élevé eu égard au problème à résoudre. Un mélange économique a été proposé par l'ITCF (Jouy et Leveau, 1992). II doit être réalisé par l'agriculteur et appliqué en post-levée. II est composé de Challenge 600 (aclonifen) et de Basagran (bentazone) mélangés volume à volume. Compte tenu du dosage des matières actives dans les formulations, l'aclonifen et la bentazone sont apportés à 300 et $240 \mathrm{~g} \mathrm{ma} \mathrm{ha}^{-1}$ (rapport pondéral 1,25). Ce mélange est bien adapté pour détruire les jeunes plantes de $G$ aparine, Sinapis arvensis et
Fallopia convolvulus dans un cadre d'emploi délimité. En dépit de ses performances agronomiques, les raisons de son efficacité ne sont pas connues.

Les 2 matières actives agissent sur des cibles différentes. L'aclonifen, un diphényléther, inhibe la protoporphyrinogène oxydase chloroplastique (Matringe et al, 1989). La bentazone inhibe la photosynthèse (Mine et Matsunaka, 1975), découple les phosphorylations oxydatives (Retzlaff et Berghaus, 1988), inhibe la fermeture des stomates (Taylor et al, 1980 ; Dunleavy et Cobb, 1984) et l'activité de l'ATPase du plasmalemme (Couderchet et Retzlaff, 1991). Ces matières actives produisent surtout des nécroses foliaires.

L'aclonifen entre rarement dans les mélanges commerciaux. Son association à l'oxadiazon pour désherber le tournesol est un cas isolé (Morvan et Petat, 1992). La bentazone participe en revanche à plusieurs mélanges homologués. Outre les mélanges cités plus haut, elle peut être associée à l'atrazine, au bromoxynil pour le désherbage du maïs (Gerwick, 1988) et au dichlorprop pour le désherbage des céréales (Brochard et Beaudoin, 1992).

Le plus souvent, la bentazone est mélangée en extemporané à d'autres herbicides ou à des insecticides. Elle peut voir son activité modifiée ou peut changer l'activité du pesticide qui lui est associé. Un effet inattendu impliquant une synergie a été rapporté pour des mélanges associant la bentazone au mefluidide, un régulateur de croissance (Rao et Harger, 1981), ou à des insecticides tels que le malathion, le parathion ou le diazinon (Campbell et Penner, 1982 ; Blumhorst et Kapusta, 1987). Dans le premier exemple, les adjuvants du mefluidide provoquent une pénétration accrue de la bentazone. Quant aux insecticides, ils interviennent en inhibant la désactivation métabolique de la bentazone dans les plantes. De même, certains mélanges à base de bentazone et d'un acide phénoxypropionique peuvent donner des synergies sur les espèces adventices des céréales sans endommager la culture (Hammann, 1995). Cependant le fait de mélanger la bentazone à un autre herbicide aboutit parfois à une réduction d'activité de ce dernier. La bentazone réduit l'activité du sethoxydime, de l'haloxyfop-methyl, du diclofop, du fluazifop, du quizalofop, du paraquat, du diquat, du triclopyr, du picloram, du TCA et de l'amitrole (Gerwick, 1988 ; Wanamarta et al, 1989 ; Aguero-Alvarado et al, 1991 ; Grichar, 1991). Le mécanisme de l'antagonisme n'est pas expliqué 
dans sa totalité. La bentazone diminue la pénétration de l'herbicide qui l'accompagne (Gerwick, 1988 ; Wanamarta et al, 1989). Mais l'espèce cible est aussi impliquée dans l'interaction puisqu'un antagonisme intervient avec le paraquat dans le cas de Phaseolus vulgaris et une synergie avec Datura sanguinalis et Setaria ptycanthum (Wichert et al, 1989 ; Wehtje et al, 1992).

Évaluer l'efficacité d'un mélange conduit à établir si les matières actives agissent de manière indépendante, si l'une modifie l'efficacité de l'autre ou si elles s'influencent mutuellement. Les modèles sont essentiels dans l'évaluation de l'action combinée. Ils permettent de calculer l'effet qui est attendu en l'absence d'effet physico-chimique ou biologique d'une matière active sur l'autre, et de mettre en évidence des synergies et des antagonismes. Les différents modèles peuvent être ramenés à 2 types. Ceux qui calculent des additivités d'effets et ceux qui calculent des additivités de doses. Le MSM (multiplicative survival model) est le modèle le plus connu permettant de calculer une additivité d'effets. II suppose que les activités sont différentes et qu'elles interviennent d'une manière séquentielle (Gowing, 1960). Si la croissance des plantes traitées est exprimée en fonction de celle des plantes non traitées, sous la forme d'un rapport, l'additivité correspond au produit des effets des matières actives administrées seules (Colby, 1967). Flint et al (1988) ont fait remarquer que la transformation logarithmique de la formule de Colby avait l'expression d'une analyse factorielle et qu'il était possible de tester l'effet d'un mélange par rapport à l'additivité. En revanche, I'ADM (additive dose model) additionne des doses. II spécifie qu'en cas d'additivité, chaque matière active d'un mélange peut être remplacée en partie ou en totalité par une dose biologiquement équivalente de l'autre, sans modifier l'effet final (Morse, 1978). L'additivité de doses nécessite que soient établies les courbes de réponse des produits appliqués seuls. L'EQM (equivalent model) est une variante de l'ADM (Green et Streibig, 1993).

Étant donné que le mélange Challenge $600+$ Basagran présente une bonne efficacité sur gaillet, des essais biologiques ont été entrepris en conditions contrôlées pour analyser les mécanismes mis en jeu. Plusieurs objectifs étaient visés : i) déterminer l'efficacité des matières actives et des produits formulés; ii) comparer 2 méthodes d'évaluation des actions combinées ; iii) évaluer l'action combinée des mélanges ; iv) dégager des éléments pouvant expliquer l'efficacité des produits formulés appliqués en mélange.

\section{MATÉRIEL ET MÉTHODES}

\section{Produits formulés et matières actives}

Les produits formulés sont les produits du commerce. Challenge 600 homologué comme herbicide de prélevée est une suspension concentrée d'aclonifen $\left(600 \mathrm{~g} \mathrm{I}^{-1}\right)$. Basagran homologué comme herbicide de post-levée est une solution de bentazone $\left(480 \mathrm{~g}^{-1}\right)$. L'aclonifen (pureté 95\%) provenait de la société Rhône-Poulenc. La bentazone (pureté 97\%) était fournie par la société Cluzeau.

\section{Matériel végétal}

Le gaillet Galium aparine L constitue le matériel végétal des expériences. Les graines provenaient de récoltes faites autour de la station. On réalisait la germination des graines en boîte de Petri, en présence de $\mathrm{KNO}_{3} 0,01 \mathrm{M}$ et à l'obscurité pendant $5-8 \mathrm{j}$. Cinq plantules étaient repiquées par pot $(300 \mathrm{ml})$. Chacun contenait un mélange de terre (argile 33\%, matière organique $2 \%$ ) et de sable calcaire, apportés volume à volume. La culture était conduite en chambre de culture : lumière $200 \mathrm{mE} \mathrm{m}^{-2} \mathrm{~s}^{-1}$ homogène sur la surface utilisée, photopériode $16 \mathrm{~h}$, humidité relative $60-90 \%$, températures différentes selon le plan expérimental comme indiqué plus loin. Une sem avant les traitements, on réduisait le nombre des plantes à 3 ou 4 par pot, et on ne retenait que les pots qui présentaient des plantes ayant 2 verticilles développés.

\section{Traitements}

Les produits formulés étaient administrés en pulvérisation foliaire avec une rampe mobile équipée de 2 buses à fente (type Albuz $110^{\circ}$ ). Pression et volume étaient réglés à $300 \mathrm{kPa}$ et $150 \mid \mathrm{ha}^{-1}$. On utilisait un autre vecteur et un autre équipement pour les matières actives. Elles étaient dissoutes dans un mélange acétone-eau ( $7-3 ; v-v)$ et pulvérisées sous la forme d'un brouillard dans une tourelle fixe équipée d'un plateau tournant. Une pression de $25 \mathrm{kPa}$ et un volume de $8 \mathrm{ml}$ constituaient les éléments de base pour un traitement comprenant 4 pots. Environ $22 \%$ de la matière active atteignait le plateau. Les plantes témoins recevaient un volume équivalent du mélange acétone-eau.

Chaque dose était administrée à 8-12 pots selon les expériences. Le jour du traitement, on prélevait les organes aériens d'un échantillon de plantes non traitées. Deux heures après le traitement, les plantes étaient replacées en chambre de culture. Quinze jours plus tard, on récoltait la partie aérienne des plantes traitées et celle d'un second lot de plantes témoins (stade 5-6 verticilles et 4 niveaux de ramification). On déterminait la croissance des plantes à partir des 
mesures de la matière sèche (MS). Les expériences étaient répétées au moins une fois.

\section{Plan expérimental permettant de calculer une additivité d'effets}

Le plan expérimental correspondait à celui d'une analyse factorielle. Les produits étaient appliqués seuls et en mélange selon un plan croisé complet. Les doses des produits appliqués seuls avaient une distribution arithmétique. Celles des mélanges du plan croisé reprenaient les doses des produits appliqués seuls. Les doses non nulles étaient $60,120,180,240,300$, $360 \mathrm{~g} \mathrm{ma} \mathrm{ha}^{-1}$ pour Challenge $600 ; 48,96,144,192$, $240,288 \mathrm{~g} \mathrm{ma} \mathrm{ha}^{-1}$ pour Basagran, 6,8, 10,1, 13,5, $16,9,20,3 \mathrm{~g} \mathrm{ha}^{-1}$ pour l'aclonifen et $5,4,8,1,10,8$, $13,5,16,2 \mathrm{~g} \mathrm{ha}^{-1}$ pour la bentazone. Produits formulés et matières actives se trouvaient dans le rapport 1,25 dans la diagonale des tableaux. La culture était conduite à $17-10^{\circ} \mathrm{C}$, températures des périodes lumineuse et obscure. Selon cette méthode, les résultats traduisent la croissance des plantes après le traitement. Ils sont exprimés en pourcentage des plantes non traitées récoltées en fin d'expérience dont le poids moyen détermine le 100\%.

Selon Colby (1967), l'action combinée des 2 herbicides est additive si la croissance de la plante traitée avec un herbicide $\mathrm{A}$ à la dose $i$, plus un herbicide $\mathrm{B}$ à la dose $j$ peut être prédite par:

$$
P_{i j}=\mathrm{A}_{i o} \mathrm{~B}_{o j} / \mathrm{AB}_{o o}
$$

avec $i, j \neq 0$; et où $P_{i j}$ représente la valeur prédite pour un mélange, $A_{i o}$ et $B_{o j}$ la croissance des plantes traitées avec les herbicides $A$ et $B$ appliqués seuls aux doses $i$ et $j$, et $\mathrm{AB}_{\text {oo }}$ la croissance de la plante témoin. II y a additivité quand $T_{i j}$ (poids des plantes traitées d'une combinaison en fin d'expérience) $=P_{i j}$.

En suivant les recommandations de Flint et al (1988), la transformation logarithmique de la formule de Colby devient :

$$
\operatorname{lot}\left(T_{i j}\right)-\log \left(\mathrm{A}_{i o}\right)-\log \left(\mathrm{B}_{o j}\right)+\log \left(\mathrm{AB}_{o o}\right)=0
$$

Le membre de gauche peut être considéré comme une interaction issue de 4 traitements dans une analyse factorielle $2 \times 2$. Elle s'écrit :

$$
l_{i j}=m_{i j}-m_{i o}-m_{o j}+m_{o o}
$$

où les différents $m$ représentent les moyennes paramétriques estimées des données transformées. L'additivité correspond à une somme algébrique nulle. La signification des interactions était testée avec un test $t$ en prenant $p \leq 0,05$. Un résultat négatif significatif traduisait une synergie et un résultat positif un antagonisme. Les calculs étaient conduits avec SAS à l'aide du programme publié par Flint et al (1988). L'erreur résiduelle ${ }^{1 / 2} /$ répétitions $^{1 / 2}$ donnait la variabilité globale sur les moyennes.

\section{Plant expérimental permettant de calculer une additivité de doses}

Une autre façon de déterminer l'efficacité des produits consiste à établir les courbes de réponse des produits appliqués seuls et en mélange (Finney, 1979). Une distribution géométrique des doses permet d'obtenir des courbes de réponse complètes. Les doses non nulles étaient $6,24,48,96,192,384,768,1536,6144$ g ma ha $^{-1}$ pour Challenge $600 ; 4,8,19,2,38,4,77$, $154,307,614,1229,4915 \mathrm{~g} \mathrm{ma} \mathrm{ha}^{-1}$ pour Basagran ; $10,8,43,2,86,173,346,691,1382,2765,11059 \mathrm{~g}$ ma ha-1 pour les produits formulés en mélange (rapport 1,25); 0,27, 1,1, 2,2, 4,3, 8,6, 17,3, 34,6, 69, 138, 276 $\mathrm{g}$ ha $^{-1}$ pour l'aclonifen et la bentazone ; et $0,6,2,4$, $4,9,9,72,19,038,9,77,7,155,311,622 \mathrm{~g} \mathrm{ha}^{-1}$ pour les matières actives en mélange (rapport 1,25). Les températures de culture étaient respectivement 20 et $15^{\circ} \mathrm{C}$ pendant les périodes lumineuse et obscure. Les résultats représentent le poids de la matière sèche (MS) des parties aériennes.

À la suite d'un traitement avec un herbicide (i), une courbe de réponse était ajustée aux données observées, en faisant appel à la fonction logistique à 4 paramètres décrite par Jensen et Kudsk (1988) :

$$
U^{\lambda}=\left(\left(D_{i}-C_{i}\right) /\left(1+\exp \left(-2\left(a_{i}+b_{i} \log x\right)\right)\right)+C\right)^{\lambda}
$$

où $U$ représente la matière sèche calculée, $D_{i}$ et $C_{i}$ les asymptotes supérieure et inférieure correspondant à la dose nulle et aux doses élevées, $a_{i}$ la position horizontale de la courbe sur l'axe des doses, $b_{i}$ la pente de la courbe au point d'inflexion, $x$ la dose d'herbicide, $\lambda$ le facteur de transformation (compris entre 0,1 et 0,9 ) permettant de minimiser la somme des carrés résiduelle. Pour les ajustements et les figures, les unités expérimentales étaient regroupées au hasard en 2 séries de données constituant 2 répétitions. Les régressions non linéaires étaient obtenues avec la méthode de plus grande vraisemblance (SAS, méthode Dud)

La relation décrite par Morse (1978) permet de calculer l'additivité des doses. Elle a pour expression

$$
z_{m}=Z_{1} Z_{2} /\left(p_{2} Z_{1}+p_{1} Z_{2}\right)
$$

où $Z_{1}, Z_{2}$ et $Z_{m}$ indiquent les doses des produits et de leur mélange donnant le même effet, et $p_{1}$ et $p_{2}$ les proportions des produits dans le mélange.

L'additivité EQM était calculée après avoir linéarisé les courbes de réponse avec une transformation logit (Green et Streibig, 1993). Elle était donnée par la relation :

$$
\begin{aligned}
Y & =p\left(a_{1}+b_{1} \log \left(x_{1}+x_{1(2)}\right)\right) \\
& +q\left(a_{2}+b_{2} \log \left(x_{2}+x_{2(1)}\right)\right)
\end{aligned}
$$

où $Y$ est la réponse du mélange exprimée en logit, et $x_{1(2)}$ et $x_{2(1)}$ les doses équivalentes de chaque herbicide exprimées dans les termes de l'autre. L'établissement des courbes de réponse complètes permet aussi de calculer une additivité des effets. 


\section{RÉSULTATS}

Le choix des températures $\left(17-10^{\circ} \mathrm{C}\right.$ pour les eśsais du type additivité d'effets) tenait compte des conditions climatiques du désherbage du pois au printemps. Mais comme la croissance des plantes n'était pas vigoureuse, les essais du type additivité de doses ont été faits à $20-15^{\circ} \mathrm{C}$. Dans ces conditions plus favorables à la croissance des plantes, les pots contenaient 3 plantes au lieu de 4. La disposition des plantes en chambre de culture dépendait du plan expérimental. Les plantes appartenant à des essais du type additivité d'effets étaient placées d'une manière aléatoire. En revanche, dans les essais du type additivité de doses, on éloignait au maximum les plantes témoins et les plantes traitées à faibles doses, des plantes traitées avec des doses élevées d'aclonifen ou de Challenge 600. En l'absence de cette règle, les plantes non traitées présentaient des nécroses du type aclonifen.

Comme indiqué, on diminuait la variabilité initiale intervenant entre les unités expérimentales en sélectionnant les pots avec le traitement. Le tri abaissait le coefficient de variation des plantes non traitées à 10,5\% le jour du traitement (effectif
$=100$ ) et à 12,4 en fin d'expérience. $\mathrm{Le} \mathrm{pH}$ de solutions de traitement variait entre 7,6 et 8,3 .

L'efficacité du mélange Challenge $600+$ Basagran $\left(300+240 \mathrm{~g} \mathrm{ma} \mathrm{ha}^{-1}\right.$ soit $0,5+0,5$ । ha-1), mise en évidence au champ sur gaillet (Jouy et Leveau, 1992) a été rerouvée en conditions contrōlées (résultat non montré). Au stade 2 verticilles, le mélange provoquait un arrêt de la croissance des plantes et leur dessèchement en une dizaine de jours. Le traitement avait un effet d'autant plus marqué que les plantes étaient jeunes (stade 1-2 verticilles). II perdait de son intensité avec l'émergence de cinquième verticille (résultat non montré).

\section{Additivité d'effets}

\section{Produits formulés}

On les a testés au cours de 2 séries d'essais. Dans une première série, les doses étaient inférieures à celles utilisées au champ. Elles étaient choisies pour produire une inhibition de croissance voisine de ou inférieure à $50 \%$, condition propice pour détecter une synergie. Challenge 600
Fig 1. Effets du Challenge 600 , du Basagran et de différents mélanges sur la croissance du gaillet. Doses inférieures à celles utilisées au champ. Poids des témoins le jour du traitement $132 \pm 23 \mathrm{mg}$ et en fin d'expérience $1016 \pm 268 \mathrm{mg}$. Erreur type 1,1\%. Réunion de 2 expériences.
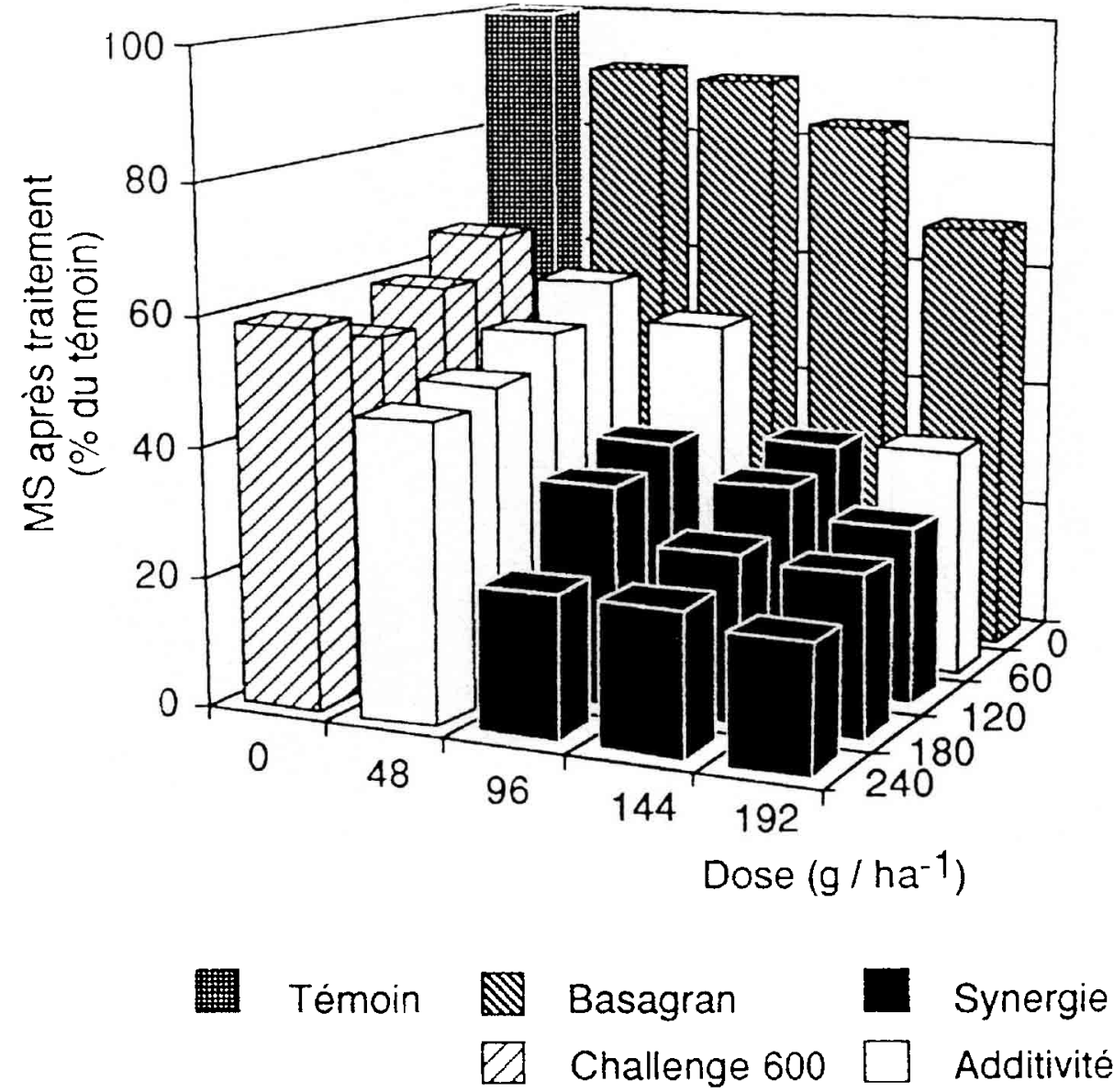
appliqué seul provoquait des nécroses claires sur les feuilles 72 heures environ après le traitement. Cet herbicide affectait la croissance du gaillet à toutes les doses, mais sans effet dose marqué (fig 1). Basagran manifestait aussi un effet de contact, mais en produisant des nécroses sombres 96 heures environ après le traitement. Les mélanges induisaient des brûlures foliaires et un éclaircissement foliaire caractéristiques du Basagran, et des décolorations apicales typiques du challenge 600. Aux doses faibles, les 2 herbicides administrés en mélange ne détruisaient pas les plantes et présentaient une efficacité du type additivité. Aux doses supérieures, les plantes subissaient un ralentissement de la croissance et étaient détruites. Dans ces combinaisons, les tests révélaient une synergie. Le mélange $120+$ $96 \mathrm{~g} \mathrm{ma} \mathrm{ha}^{-1}$ ou $0,2+0,2$ I ha-1 constituait le premier niveau impliquant une synergie et produisant un effet létal. Bien que retenant beaucoup de bouillie, les cotylédons n'étaient pas détruits.

Dans une seconde série d'essais, les doses choisies encadraient la dose préconisée au champ. L'ajout de doses plus élevées et le développement moins avancé des plantes s'est traduit par un effet prononcé des traitements (fig 2). Les mélanges appliqués à doses fortes produisaient un arrêt de croissance presque immédiat, un étranglement de la tige entre le second et le troisième verticille, et la destruction des plantes. L'implication d'une synergie était trouvée dans 8 mélanges sur 9.

\section{Matières actives}

Les matières actives appliquées seules affectaient la croissance des plantes, et appliquées en mélange pouvaient les détruire. Comme dans le cas des produits formulés, les premiers symptômes apparaissant le troisième jour après le traitement étaient ceux du diphényléther. La bentazone et les mélanges (à doses élevées) produisaient les brûlures sombres un jour plus tard. À la récolte, aucune des 2 matières actives appliquée seules ne provoquait la destruction des plantes (fig 3). Les symptômes des plantes traitées avec les mélanges dépendaient principalement de la dose de bentazone dans le mélange. Les mélanges à faibles doses de bentazone montraient des symptômes de bentazone sur les 2 premiers verticilles présents au moment du traitement, des symptômes d'aclonifen sur les 2 verticilles plus jeunes suivants et des étranglements de tige. L'augmentation des doses de bentazone

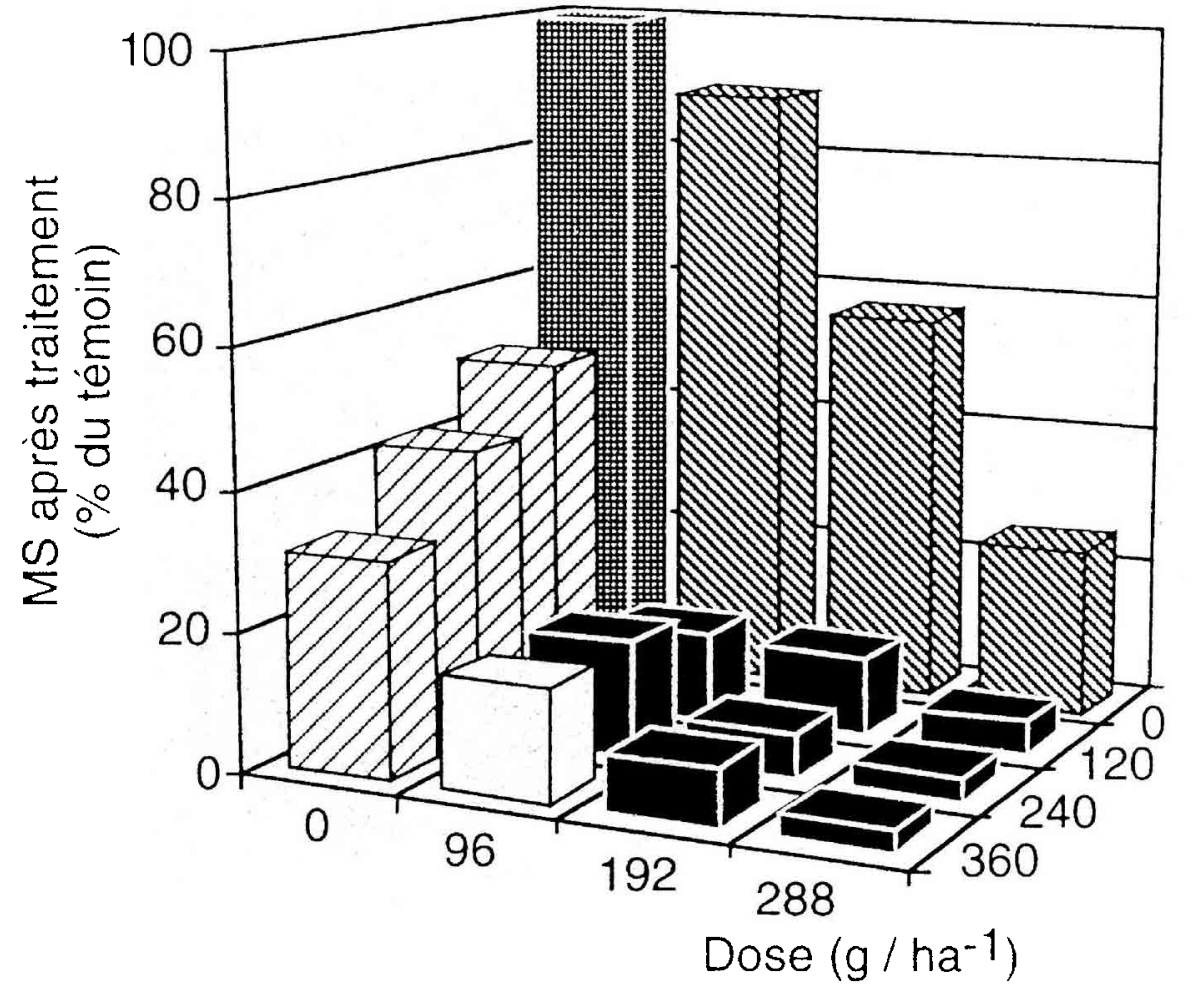

Témoin
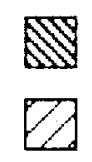

Basagran

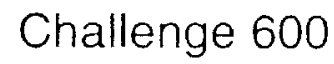

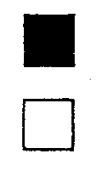

Synergie

Additivité
Fig 2. Effets du Challenge 600 , du Basagran et de différents mélanges sur la croissance du gaillet. Doses encadrant celles utilisées au champ. Poids des témoins le jour du traitement $131 \pm$ $16 \mathrm{mg}$ et en fin d'expérience $817 \pm 97 \mathrm{mg}$. Erreur type $1,2 \%$. Réunion de 2 expériences. 
Fig 3. Effets de l'aclonifen, de la bentazone et de différents mélanges sur la croissance du gaillet. La gamme des doses d'aclonifen appliqué seul était 1,25 fois supérieure à celles de doses de bentazone appliqué seul. Poids des témoins le jour du traitement $96 \pm 8 \mathrm{mg}$ et en fin d'expérience $699 \pm 77 \mathrm{mg}$. Erreur type 1,1\%.
Fig 4. Effets de l'aclonifen, de la bentazone et de différents mélanges sur la croissance du gaillet. Les doses d'aclonifen et de bentazone appliqués seuls étaient identiques. Poids des témoins le jour du traitement $123 \pm 7 \mathrm{mg}$ et en fin d'expérience $661 \pm 38 \mathrm{mg}$. Erreur type $1,3 \%$.

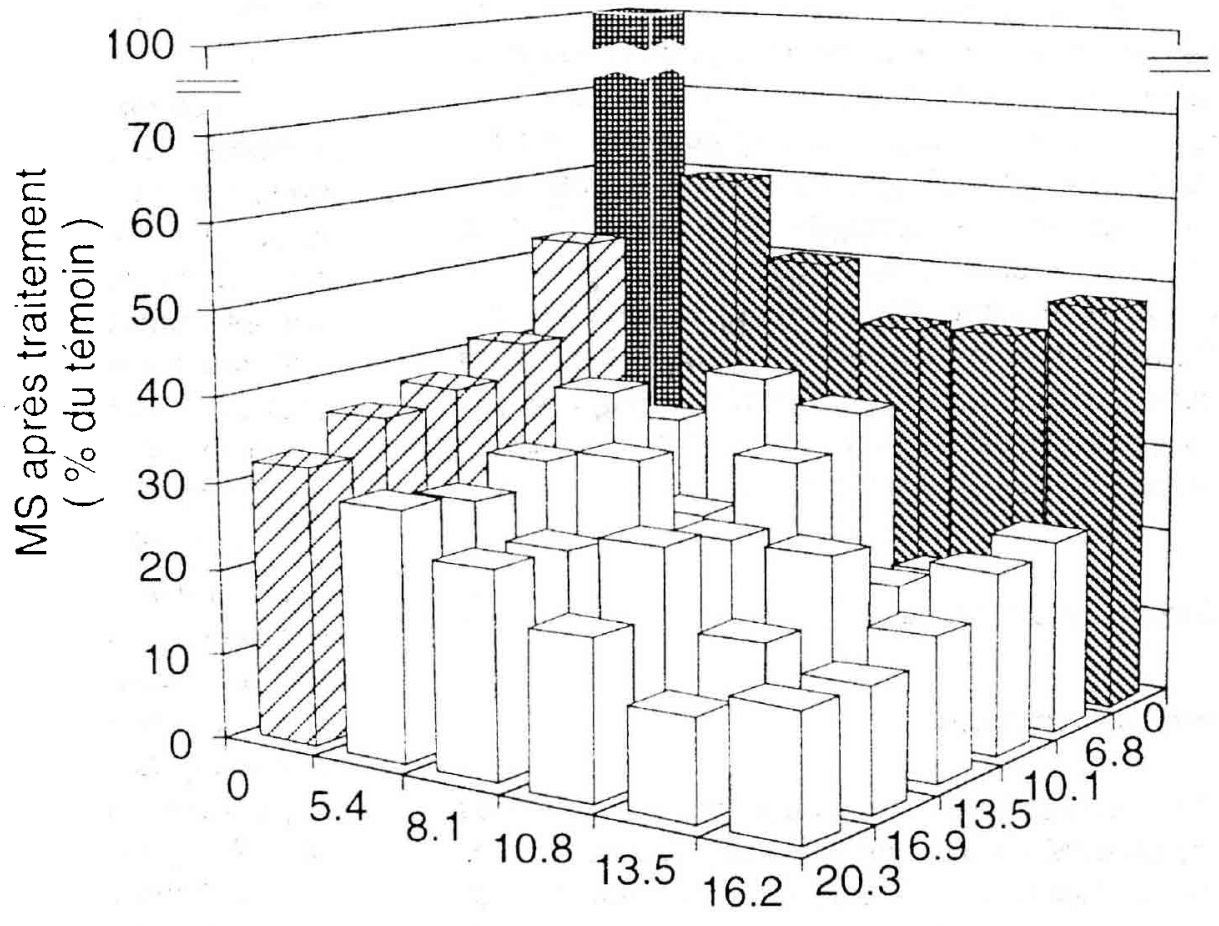

( $\mathrm{g} \mathrm{ha}^{-1}$ )

$\begin{array}{cc}\text { Témoin } & \mathbb{N} \text { bentazone } \\ \square \text { aclonifen } & \square \text { Synergie } \\ & \text { Additivité }\end{array}$

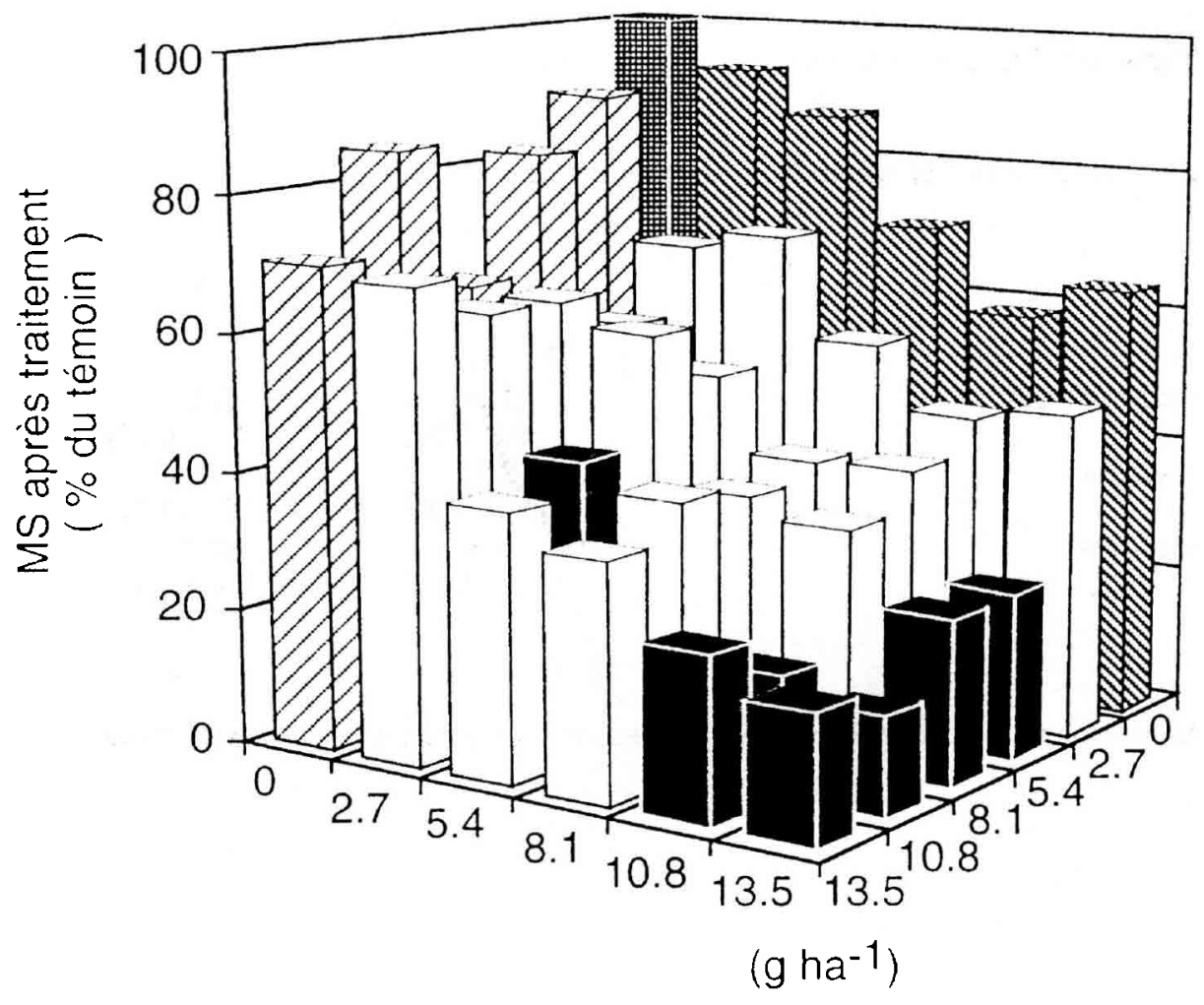

Témoin bentazone $\square$ Synergie $\square$ aclonifen $\square$ Additivité 
dans les mélanges s'accompagnait de la disparition des symptômes d'aclonifen et du développement d'une nécrose apicale. La destruction de tous les apex d'une plante se produisait avec les mélanges ayant des doses élevées de bentazone. L'analyse des résultats ne faisait apparaître ni antagonisme ni synergie (fig 3). Dans une autre expérience, les matières actives ont été mélangées selon d'autres rapports (fig 4). Sept combinaisons sur 25 montraient une synergie, l'une d'entre elles $\left(13,5+10,8 \mathrm{~g} \mathrm{ha}^{-1}\right)$ ayant le rapport de matières actives 1,25 .

\section{Additivité de doses}

\section{Produits formulés}

Challenge 600 produisait des symptômes foliaires même aux doses les plus faibles $(6 \mathrm{~g} \mathrm{ma}$ $\mathrm{ha}^{-1}$ ). Aux doses intermédiaires les symptômes étaient plus impressionnants que nuisibles et la dose maximale $6144 \mathrm{~g} \mathrm{ma} \mathrm{ha-1}$ ne provoquait pas la mort des plantes. Basagran n'affectait pas la croissance aux faibles doses (5 à $38 \mathrm{~g} \mathrm{ma}$ $\mathrm{ha}^{-1}$ ). Il détruisait quelques apex à $614 \mathrm{~g} \mathrm{ma}$ $\mathrm{ha}^{-1}$. A partir de la dose $192 \pm 154 \mathrm{~g} \mathrm{ma} \mathrm{ha}^{-1}$, le

Tableau I. Produits formulés.

a. Paramètres des ajustements avec intervalle de confiance mélange des 2 produits (rapport 1,25 ) provoquait la mort des plantes.

Les sommes de carrés résiduelles des 3 ajustements ont été rendues minimales avec une transformation logarithmique des données. Des calculs préliminaires ont indiqué aussi que les 3 courbes de réponse pouvaient être décrites avec une asymptote supérieure commune : $D=1377$ $\pm 27 \mathrm{mg}$ (tableau I). Les réponses différentes des plantes aux produits formulés appliqués seuls se traduisaient par des pentes différentes, faible pour Challenge 600 , forte pour Basagran, et intermédiaire pour le mélange (tableau I, fig 5).

En partant de l'équation des courbes de réponse, il a été trouvé que la $I_{50}$ des produits (dose provoquant une inhibition de croissance $50 \%$, correspondant à une biomasse située à égale distance des asymptotes inférieure et supérieure) était $38 \mathrm{~g} \mathrm{ma} \mathrm{ha-1}$ pour Challenge $600,201 \mathrm{~g} \mathrm{ma} \mathrm{ha}^{-1}$ pour Basagran, et $59+47 \mathrm{~g}$ ma ha-1 pour le mélange. Comme les asymptotes inférieures étaient différentes pour les 3 courbes de réponse, différents niveaux d'inhibition de croissance ont également été calculés en prenant comme référence l'asymptote inférieure de la courbe de réponse donnée par le mélange. Avec ce mode de calcul, la $I_{50}$ de Challenge 600

\begin{tabular}{|c|c|c|c|c|}
\hline & $a$ & $b$ & $C$ & $r^{2}$ \\
\hline Challenge 600 & $\begin{array}{c}1,98 \\
(1,30: 2,66)\end{array}$ & $\begin{array}{c}-0,54 \\
(-0,71 ;-0,38)\end{array}$ & $\begin{array}{c}360 \\
(316 ; 403)\end{array}$ & 0,97 \\
\hline Basagran & $\begin{array}{c}7,61 \\
(6,13 ; 9,09)\end{array}$ & $\begin{array}{c}-1,44 \\
(-1,70 ;-1,17)\end{array}$ & $\begin{array}{c}194 \\
(178 ; 209)\end{array}$ & 0,99 \\
\hline $\begin{array}{l}\text { Mélange } \\
\qquad(1,25+1)\end{array}$ & $\begin{array}{c}3,39 \\
(2,82 ; 3,97)\end{array}$ & $\begin{array}{c}-0,73 \\
(-0,84 ;-0,62)\end{array}$ & $\begin{array}{c}160 \\
(145 ; 175)\end{array}$ & 0,99 \\
\hline
\end{tabular}

b. Inhibition de croissance calculée par rapport à l'asymptote inférieure du mélange (dose en $g$ ha $^{-1}$ )

$\begin{array}{lcccc} & 50 \% & 75 \% & 85 \% & 91,5 \% \\ \text { Challenge } 600 & & & & - \\ \text { Basagran } & 55 & 283 & 394 & 527 \\ \text { Mélange : Challenge 600 + Basagran } & 59+47 & 126+101 & 195+156 & 300+240 \\ \left(I h a^{-1}\right) & (0,1+0,1) & (0,2+0,2) & (0,32+0,32) & (0,5+0,5)\end{array}$



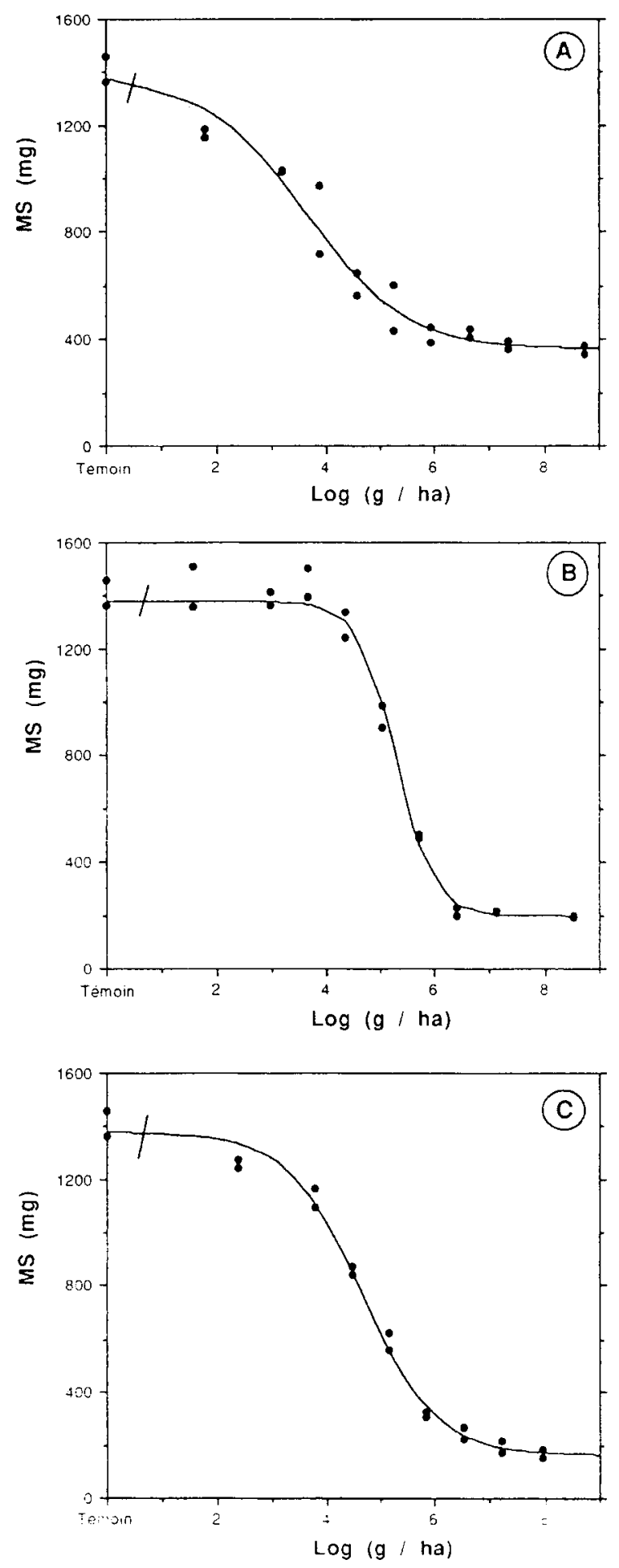

Fig 5. Courbes de croissance du gaillet traité avec les produits formulés, $\mathbf{A}=$ Challenge $600, \mathbf{B}=$ Basagran, $\mathrm{C}=$ mélange Challenge $600+$ Basagran $(1,25+1)$.

passe de 38 à $55 \mathrm{mg} \mathrm{ma} \mathrm{ha-1} \mathrm{mais} \mathrm{reste} \mathrm{bien}$ inférieure à celle du Basagran (tableau I). II apparaît aussi que le mélange $0,5+0,5$ I ha-1 induit une inhibition de croissance de $91,5 \%$.

La position relative des 3 courbes de réponse sur l'axe des doses, liée aux valeurs du para- mètre $a_{i}$, donne une lecture directe des efficacités relatives, c'est-à-dire des rapports de doses donnant le même effet (tableau I, fig 6). Au voisinage des $I_{50}$, l'efficacité du Challenge 600 est supérieure à celle du mélange (efficacité relative 2,77 ), elle même supérieure à celle du Basagran (efficacité relative 1,89 ). Mais les efficacités relatives changent à toutes les doses, les courbes n'étant pas parallèles. Le mélange constitue le traitement le plus efficace à partir du niveau d'inhibition $75 \%$ environ. Challenge 600 et Basagran présentent la même efficacité à l'intersection $\mathrm{A}$ ( $314 \mathrm{~g} \mathrm{ma} \mathrm{ha}^{-1}$; inhibition de croissance $77,5 \%$ ). La courbe du mélange croise celle du Challenge 600 en B (202 g ma ha-1 et $90+112 \mathrm{~g} \mathrm{ma} \mathrm{ha}^{-1}$; inhibition de croissance $75,3 \%$ ) puis rejoint celle du Basagran en $\mathrm{C}$ (environ $493 \mathrm{~g} \mathrm{ma} \mathrm{ha}^{-1}$ et $274+219 \mathrm{~g} \mathrm{ma} \mathrm{ha}^{-1}$; inhibition de croissance $93 \%)$. La dose du mélange préconisé par l'ITCF

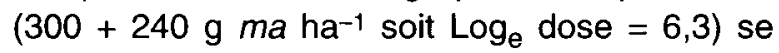
situe dans cette zone.

La courbe d'additivité ADM est toujours positionnée entre les courbes de réponse induites par Challenge 600 et Basagran, et passe par A (fig 6). Elle est à gauche de la courbe du mélange pour les faibles doses, révélant un antagonisme, l'intercepte au niveau d'inhibition de croissance $68 \%\left(87+69 \mathrm{~g} \mathrm{ma} \mathrm{ha}^{-1}\right)$, puis se situe à droite révélant une synergie aux doses élevées. La courbe d'additivité MSM est presque confondue à celle de l'ADM aux faibles doses et coupe la courbe du mélange au niveau d'inhibition $61 \%$ $\left(68+55 \mathrm{~g} \mathrm{ma} \mathrm{ha}^{-1}\right)$. Cependant elle ne passe pas par $A$ et intercepte à nouveau la courbe du mélange au niveau d'inhibition de croissance $95 \%$ environ. Comme l'additivité ADM, l'additivité MSM montre un antagonisme aux faibles doses puis une synergie, limitée à l'intervalle $(91+73$; $274+219 \mathrm{~g} \mathrm{ma} \mathrm{ha}^{-1}$; inhibition de croissance $61-95 \%$ environ).

\section{Matières actives}

Les doses élevées de matières actives appliquées seules $\left(276 \mathrm{~g} \mathrm{ha}^{-1}\right)$ provoquaient la destruction des plantes. Une transformation logarithmique des données produisait les meilleurs ajustements dans le cas de l'aclonifen et de la bentazone. Quant au mélange, la meilleure solution était obtenue en prenant $\lambda=0,3$. Comme les 3 types de traitements avaient les mêmes plantes témoins, que tous provoquaient la mort des plantes aux doses élevées, et que les ajustements indépendants donnaient des asymptotes inférieure et supérieure comparables, on a réali- 


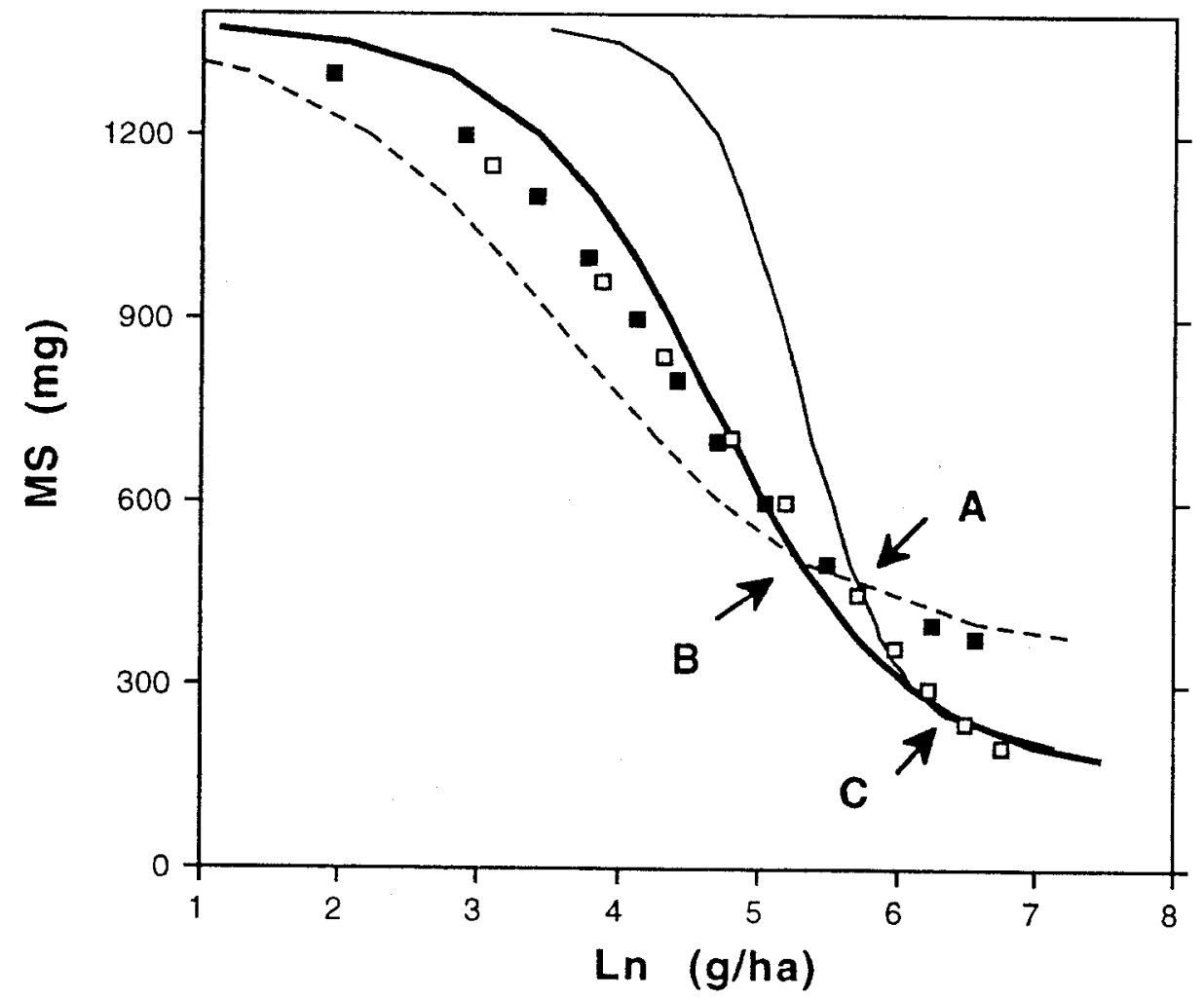

Fig 6. Courbes de croissance ajustées du gaillet traité avec les produits formulés, -.-- Challenge 600, --Basagran, --- mélange Challenge $600+$ Basagran $(1,25+1), \mathbf{\square}$ additivité ADM, additivité MSM.

sé les ajustements simultanés en prenant des asymptotes communes aux 3 traitements (tableau II et fig 7). Les valeurs trouvées pour $C$ et $D$ sont voisines de celles de la biomasse des plantes témoins récoltées lors du traitement $(156 \pm 16$ $\mathrm{mg}$ ) et en fin d'expérience (1 $675 \pm 179 \mathrm{mg}$ ).

Les pentes des courbes de réponse sont très différentes pour l'aclonifen et la bentazone, celle

Tableau II. Matières actives.

a. Paramètres des ajustements avec intervalle de confiance

$a$

Aclonifen

Bentazone

Mélange

$(1,25+1)$
$(0,71 ; 0,90)$

1,62

$(1,32 ; 1,92)$

1,06

$(0,49 ; 1,64)$
$-0,32$

$(-0,34 ;-0,29)$

$-0,82$

$(-0,93 ;-0,71)$

$-0,61$

$(-0,82 ;-0,39)$ $r^{2}$

0,99

0,99

0,90

b. Inhibition de croissance (doses en $\mathrm{g} \mathrm{ha}^{-1}$ )

$50 \%$

$75 \%$

71,53

14,16

7,24
$85 \%$

$95 \%$

194,74

20,87
1308,82

43,68

Bentazone

$7,96+6,36$

$13,44+10,76$

$36,49+29,20$ 

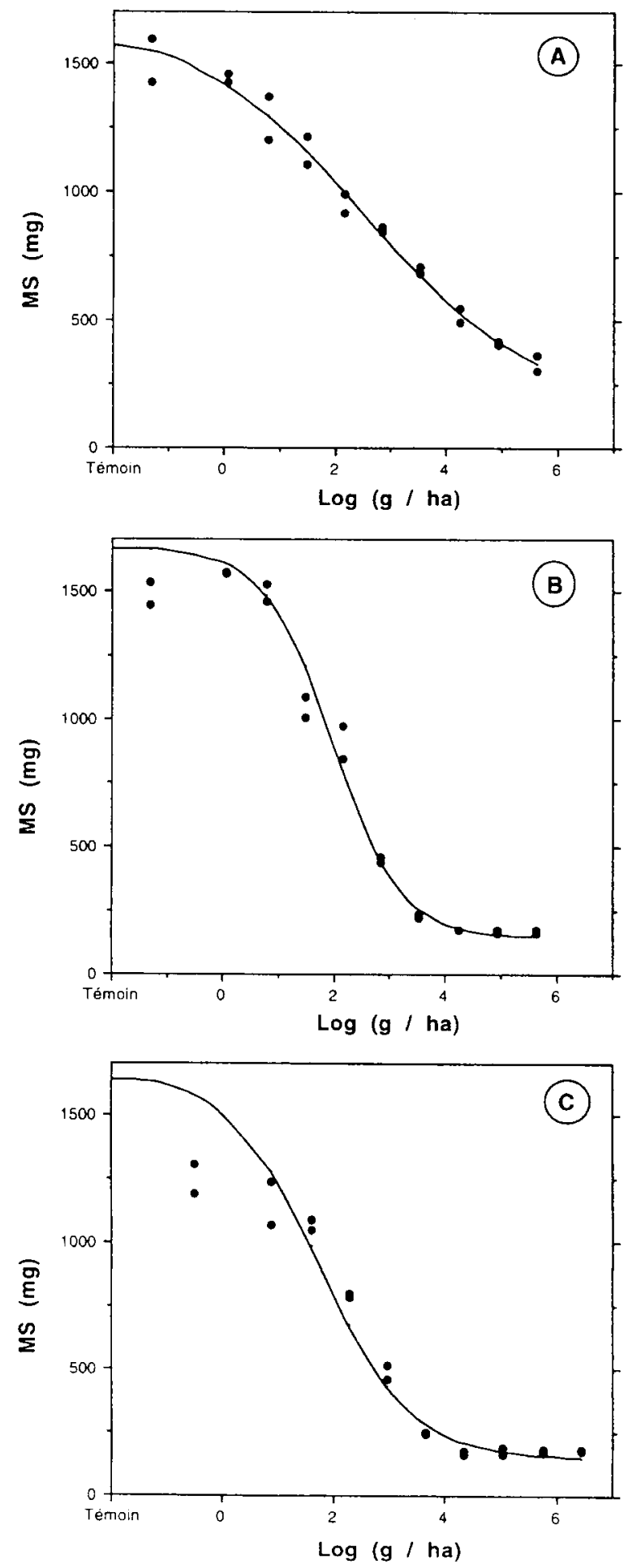

Fig 7. Courbes de croissance du gaillet traité avec les matières actives. $\mathbf{A}=$ aclonifen, $\mathbf{B}=$ bentazone, $\mathbf{C}=$ mélange aclonifen + bentazone $(1,25+1)$

du mélange étant intermédiaire (tableau II). Les différences vont dans le sens de celles observées pour les produits formulés. En revanche, les valeurs du paramètre $a_{i}$ présentent des écarts moins importants, ce qui se traduit par un positionnement plus serré des courbes sur l'axe des doses (fig 8). L'aclonifen est plus efficace que la bentazone aux faibles doses seulement, c'est-àdire pour des niveaux d'inhibition inférieurs à $36 \%$, limite déterminée par $A^{\prime}$ (fig 8). Pour une inhibition $50 \%$, l'efficacité relative de la bentazone par rapport à l'aclonifen est 1,75 puis augmente à 5,$0 ; 9,3 ; 30,0$ pour des niveaux d'inhibition 75 , 85 et $95 \%$. La courbe du mélange ne passe pas par $A^{\prime}$, coupe la courbe définie par l'acionifen en $\mathrm{B}^{\prime}\left(2,44 \mathrm{~g}\right.$ aclonifen ha- $\mathrm{A}^{-1} ; 1,36+1,08 \mathrm{~g} \mathrm{ha}^{-1}$; inhibition de croissance $26 \%$ ) et celle de la bentazone en $\mathrm{C}^{\prime}(13,7 \mathrm{~g}$ bentazone ha-1; $1,45+1,16 \mathrm{~g}$ ha $^{-1}$; inhibition de croissance $74 \%$ ). Entre $B^{\prime}$ et $C^{\prime}$, la courbe du mélange se trouve à l'extérieur des 2 aires définies par les matières actives. Selon le modèle d'additivité de doses, le segment $\mathrm{B}^{\prime} \mathrm{C}^{\prime}$ traduit une synergie pour tous les mélanges de ces 2 matières actives. Pour le rapport de mélange 1,25 , le modèle d'additivité de doses établit qu'il y a synergie pour une gamme de doses allant de $13+10,4 \mathrm{~g} \mathrm{ha}^{-1}$ aux doses élevées, c'est-à-dire pour une gamme plus grande que celle définie par le modèle d'additivité des effets $\left(2,38+1,90 ; 18,04+14,43 g\right.$ ha $\left.^{-1}\right)$.

Comme les courbes de réponses aux 3 traitements avaient des asymptotes communes, il était possible d'évaluer l'action jointe des matières actives par rapport à l'additivité EQM. En suivant le mode de calcul décrit par Green et Streibig (1993), le mélange était représenté par une droite, et l'additivité par une courbe (fig 9). Une synergie était trouvée dans l'intervalle de doses $0,63+0,50 ; 91,12+72,90 \mathrm{~g} \mathrm{ha}^{-1}$.

\section{DISCUSSION}

Le premier objectif du travail était de comparer l'efficacité de 2 matières actives appliquées avec ou sans formulation. À effet égal, les doses de matières actives dissoutes dans le mélange acétone-eau étaient toujours plus faibles que celles des matières actives présentes dans leur formulation commerciale. Lors des traitements, les pulvérisations faites avec le mélange acétone-eau donnaient des dépôts fins à toutes les doses. En revanche Challenge 600 , dont la formulation est une suspension concentrée destinée à des traitements de pré-levée, donnait des dépôts plus larges. Avec cet herbicide, la qualité de la couverture se détériorait quand les doses augmentaient (6 $\left.144 \mathrm{~g} \mathrm{ha}^{-1}\right)$. Toutefois, la différence concernant les vecteurs de pulvérisation n'explique pas tout puisque la différence d'efficacité entre les matières actives et les produits formulés touche plus Basagran que Challenge 600 (figs 6 et 8 ). 


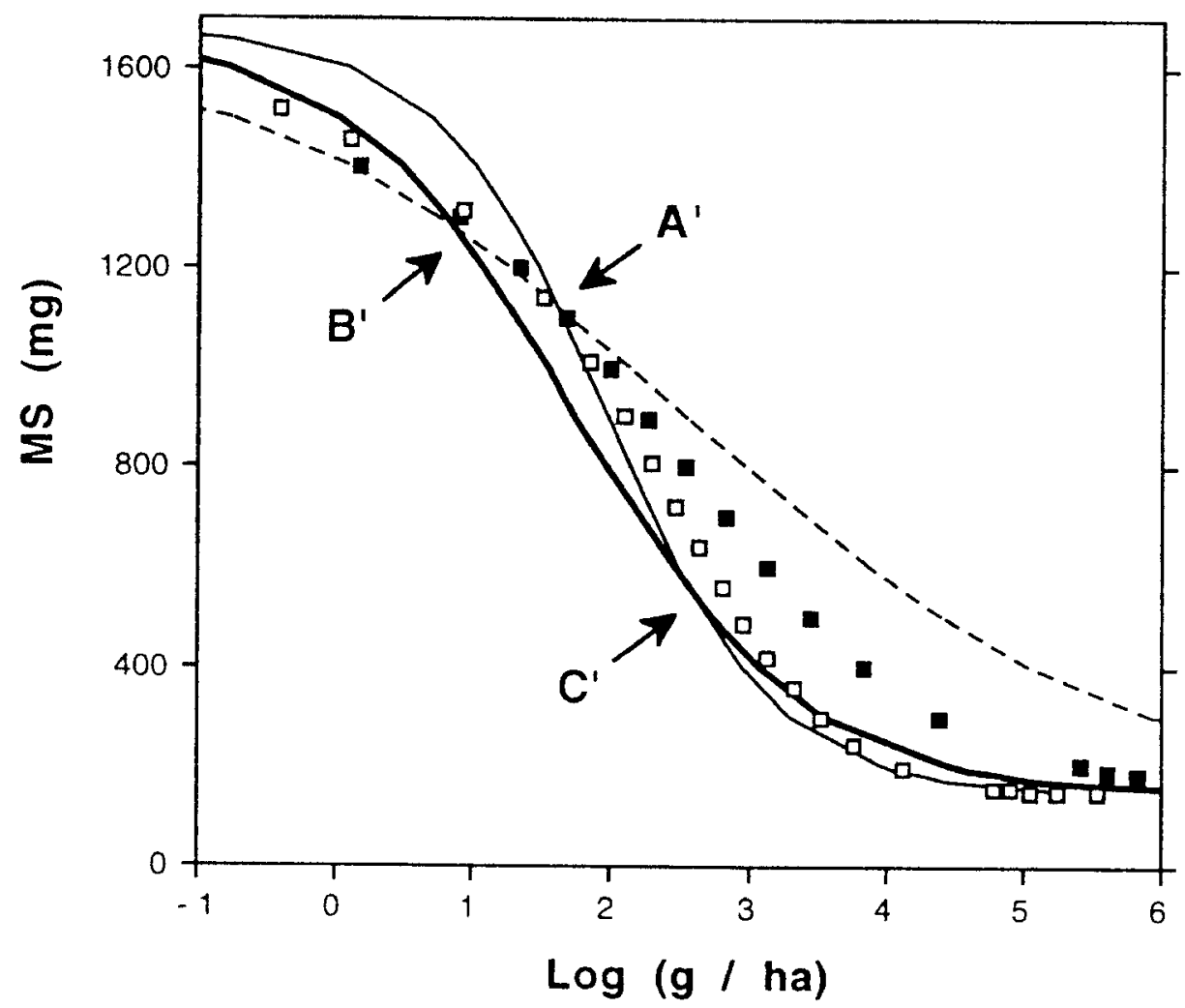

Fig 8. Courbes de croissance ajustées du gaillet traité avec les matières actives, .... aclonifen, bentazone, --- mélange aclonifen + bentazone $(1,25$ +1 ), $\square$ additivité ADM, $\square$ additivité MSM.

Les symptômes d'aclonifen et de bentazone n'étaient pas trouvés sur les mêmes organes. L'aclonifen produisait des symptômes sur les différentes parties aériennes touchées par la pulvé- risation, mais d'une manière d'autant plus marquée que les feuilles étaient en croissance. En revanche l'action de la bentazone se limitait aux verticilles bien développés. Quand les 2 matières

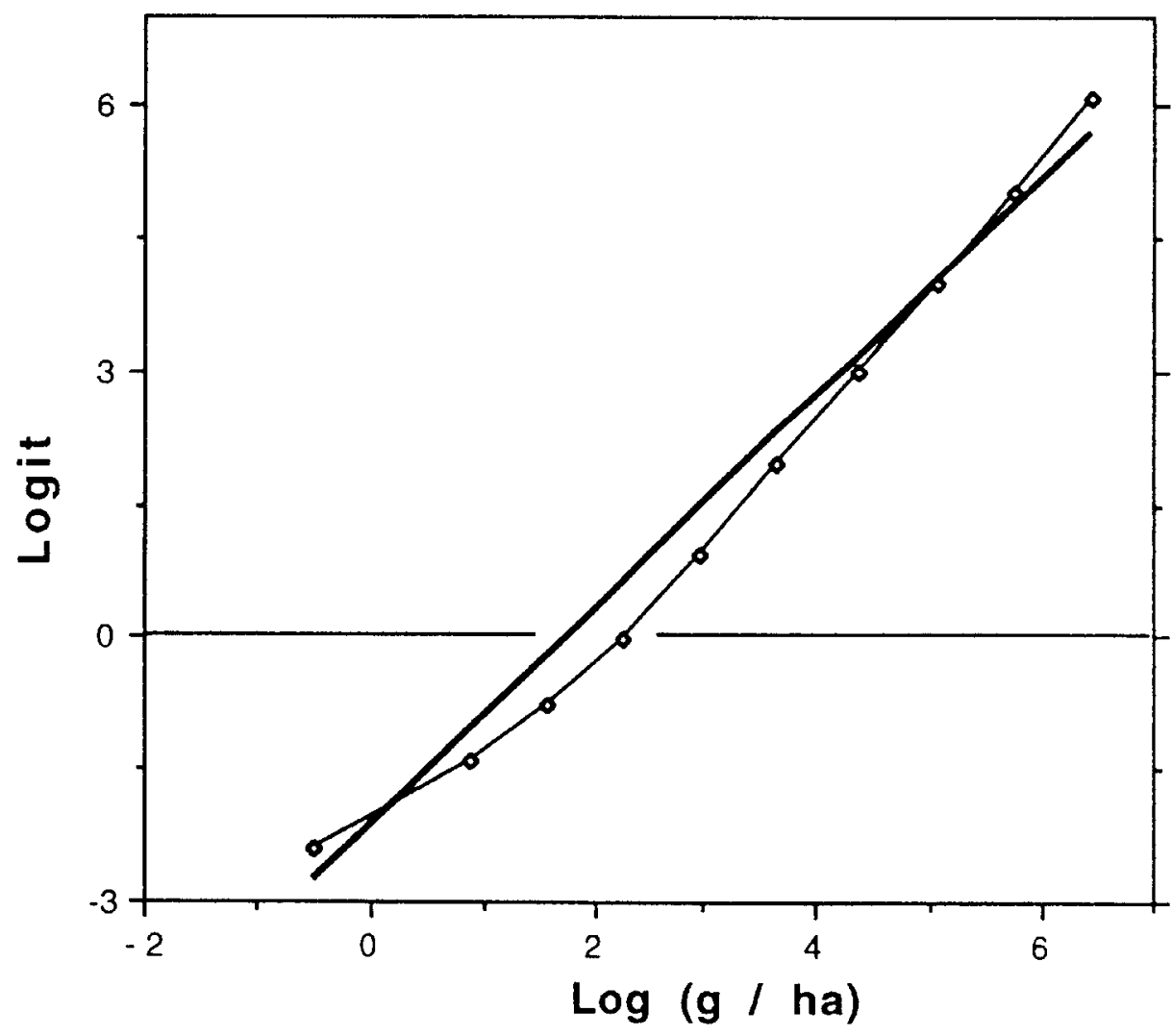

Fig 9. Courbe d'inhibition de croissance du gaillet, traité avec le mélange des matières actives, linéarisée par une transformation logit. additivité EQM. 
actives étaient en mélange, on observait la juxtaposition des 2 types de symptômes. Cette juxtaposition des symptômes pourrait être reliée au mode d'action des 2 matières actives. Comme l'aclonifen interfère avec la synthèse des chlorophylles, alors que la bentazone inhibe pour une part la photosynthèse, une hypothèse serait que l'aclonifen affecterait principalement les organes chlorophylliens en voie de développement tandis que la bentazone toucherait surtout les feuilles ayant une photosynthèse active. Mais une distribution différente des symptômes pourrait être aussi la conséquence d'une différence de pénétration des matières actives selon l'âge des organes. En l'absence de données expérimentales comparant les cinétiques de pénétration foliaire de l'aclonifen et de la bentazone, la distribution différente de leurs symptômes est encore mal comprise.

Le second objectif du travail était de comparer les 2 méthodes qui utilisent des plans expérimentaux différents et 2 types de modèles. Le plan factoriel offre l'avantage d'analyser des mélanges ayant différents rapports de matières actives et est assorti d'une méthode d'analyse rapide. Mais il présente 2 inconvénients : i) un nombre élevé de doses par matière active appliquée seule $(>8)$ engendre un plan croisé qui est lourd expérimentalement, et un nombre réduit de doses risque de donner des effets éloignés de l'inhibition de croissance $50 \%$; ii) les données ne peuvent être évaluées que par le modèle d'additivité d'effets (MSM). Par comparaison, le plan expérimental mettant en œuvre des courbes de réponse et exigeant une gamme étendue de doses conduit à n'étudier qu'un nombre limité de rapports différents de matières actives. Mais il permet de calculer différents niveaux d'inhibition de croissance, des efficacités relatives, et d'évaluer l'efficacité des mélanges avec les 2 modèles.

Le modèle d'additivité d'effets (MSM) a souvent été cité dans la littérature car, pour évaluer une combinaison, il suffit de connaître la croissance du témoin, les effets des produits appliqués seuls et l'effet du mélange. Mais il suppose que les 2 matières actives agissent de manière séquentielle. II est nécessaire de calculer des pourcentages de croissance à l'aide d'un 100\% inconnu. Enfin le MSM ne redonne pas la courbe de réponse d'un produit quand il est mélangé avec lui-même (Green et al, 1995). Le modèle d'additivité de doses (ADM) ne fait pas de restrictions quant au mode d'action des matières actives et permet de retrouver la courbe de réponse d'un produit quand il est mélangé avec lui-même (Green et al, 1995).

Les données expérimentales établies dans ce travail ne permettent pas de comparer les 2 méthodes en toute rigueur puisque les 2 méthodes ont été mises en œuvre à des températures différentes. II est cependant peu probable que cette différence puisse expliquer à elle seule le surplus d'information apportée par la méthode des courbes de réponse, ajustées avec la technique de la régression et analysées avec le modèle d'additivité de doses. En faisant appel à une large gamme de doses, cette méthode a permis de révéler un antagonisme dans les mélanges de produits formulés et de matières actives appliqués à faibles doses. Elle seule a clairement montré l'intervention d'une synergie dans le cas des matières actives. Quant aux 2 modèles, ils ont donné des courbes d'additivité comparables aux faibles doses mais différentes aux doses élevées. Compte tenu de la remarque de Green et al (1995), le modèle d'additivité de doses constitue la meilleure référence pour évaluer les mélanges.

Le troisième point du travail se proposait d'évaluer les mélanges. Quand les matières actives ou les produits formulés sont mélangés à faibles doses, un antagonisme intervient. En revanche, aux doses élevées, l'analyse des mélanges met en évidence une synergie dont les caractéristiques sont différentes pour les produits formulés et les matières actives. Dans le cas du Challenge 600 + Basagran, la synergie se manifeste aux doses élevées provoquant toujours des niveaux inhibition de croissance supérieurs à $50 \%$. Les courbes de réponse situent la limite inférieure des doses donnant une synergie à $67,5+54,0$ et $82,5+66,0 \mathrm{~g} \mathrm{ma} \mathrm{ha}^{-1}$ respectivement pour les modèles ADM et MSM, correspondant à $0,11+0,11$ et $0,13+0,13 \mid$ ha $^{-1}$. Dans le cas des matières actives dissoutes dans le mélange acétone-eau, la synergie, qui se manifeste à dose basse, n'engendre pas forcément des effets drastiques, des niveaux d'inhibition pouvant être inférieures à $50 \%$. La synergie montrée par les matières actives résulte d'un mécanisme encore inconnu bien qu'une complémentarité de leur site d'action ait été notée. La synergie des produits formulés pourrait être la somme de plusieurs phénomènes, impliquant la synergie des matières actives et un effet croisé des formulants. Ces derniers auraient 2 effets : i) en modifiant les positions relatives des courbes de réponse des produits parents (Challenge 600 et Basagran par rapport à aclonifen et bentazone), ils limiteraient la zone de synergie aux doses éle- 
vées, très phytotoxiques et intéressantes pour le désherbage ; ii) à ces doses, ils provoqueraient une synergie plus puissante que celle mise en œuvre par les matières actives puisque la courbe du mélange des produits formulés se situe, aux doses élevées, à l'extérieur ou à la limite de l'aire délimitée par les courbes du Challenge 600 et du Basagran alors que la courbe du mélange des matières actives se situe, pour des doses équivalentes, à l'intérieur de l'aire décrite par les courbes de l'aclonifen et de la bentazone (figs 6 et 8 ). La bentazone est une matière active soluble dans l'eau. Mais quand l'eau du dépôt foliaire est évaporée, la bentazone cristallise facilement (Gerwick, 1988). Les composants de la formulation du Challenge 600 pourraient inhiber la cristallisation de la bentazone et accroître sa pénétration dans les feuilles du gaillet. II est établi que la phytotoxicité de la bentazone vis-à-vis de l'amarante est augmentée par les tensio-actifs (Manthey et al, 1992).

En conclusion, si la méthode utilisant un plan factoriel est simple de mise en œuvre et offre une analyse rapide de l'efficacité des mélanges, elle est moins performante que la méthode faisant appel à des courbes de réponse. L'efficacité des produits formulés additionnerait un effet synergique des matières actives et un effet des formulants en intensifiant la synergie et en la positionnant dans la gamme des doses très phytotoxiques au gaillet.

L'étude faite en conditions contrôlées a permis de décrire un mécanisme pour tenter d'expliquer l'efficacité du mélange Challenge $600+$ Basagran vis-à-vis du gaillet. Mais rien n'indique que les efficacités des produits appliqués seuls ou en mélange, notées en conditions contrôlées, étaient identiques à celles intervenant au champ. En particulier, en comparant nos données à celles de l'ITCF, la sensibilité des gaillets cultivés en chambre de culture paraissait plus grande que celle des plantes de plein champ vis-à-vis du Basagran. Une étude est en cours pour déterminer l'influence des facteurs de l'environnement sur l'efficacité des herbicides et sur leurs efficacités relatives.

\section{REMERCIEMENTS}

Nous remercions amicalement C Gauvrit et $\mathrm{L}$ Jouy pour leurs conseils constructifs prodigués au cours du travail. Nos remerciements s'adressent aussi à Rhône Poulenc qui a fourni l'aclonifen. Une partie du travail était financé par I'UNIP.

\section{RÉFÉRENCES}

Aguero-Alvarado R, Appelby AP, Armstrong DJ (1991) Antagonism of haloxyfop activity in tall fescue (Festuca arundinacea) by dicamba and bentazon. Weed Sci 39, 1-5

Blumhorst MR, Kapusta G (1987) Mefluidide as a enhancing agent for postemergence broadleaf herbicides in soybeans (Glycine max). Weed Technol 1, 149-153

Brochard M, Beaudoin X (1992) Nouvelles perspectives de désherbage du lin. In : XVe Conf Columa. J Intern Lutte Mauvaises Herbes, Versailles, 669-674

Campbell JR, Penner D (1982) Enhanced phytotoxicity of bentazon with organosphosphate and carbamate insecticides. Weed Sci 30, 324-326

Colby SR (1967) Calculating synergistic and antagonistic responses of herbicide combinations. Weeds $15,20-22$

Couderchet M, Retzlaff G (1991) The role of the plasma membrane ATPase in bentazone sethoxydim antagonism. Pestic Sci 32, 295-306

Dunleavy PJ, Cobb AH (1984) Bentazone-induced stomatal movement in epidermal peels from Chenopodium album L. New Phytol 97, 115-120

Finney DJ (1979) Bioassay and the practice of statistical inference. Intern Statist Rev 47, 1-12

Flint JL, Cornelius PL, Barrett M (1988) Analyzing herbicide interactions: a statistical treatment of Colby's method. Weed Technol 2, 304-309

Gerwick BC (1988) Potential mechanisms for bentazon antagonism with haloxyfop. Weed Sci 36, 286-290

Gowing DP (1960) Comments on tests of herbicide mixtures. Weeds 8, 379-391

Green JM, Streibig JC (1993) Herbicides mixtures. In : Herbicide Bioassays (Streibig JC, Kudsk P, eds), CRC Press, Boca Raton, FL, 117-136

Green JM, Jensen JE, Streibig JC (1995) Models to assess joint action of pesticide mixtures. In : Understanding Crop Protection Mixtures, aab Symposium Silsoe, Royaume-Uni, 61-68

Grichar WJ (1991) Sethoxydim and broadleaf herbicide interaction effects on annual grass control in peanuts (Arachis hypogaea). Weed Technol 5, 321-324

Hammann H (1995) Patenting mixtures. In : Understanding Crop Protection Mixtures, aab Symposium Silsoe, Royaume-Uni, 11-18

Jensen PK, Kudsk P (1988) Prediction of herbicide activity. Weed Res 28, 473-478

Jouy L, Leveau C (1992) Des possibilités performantes et économiques. Perspec Agric 175, 54-56

Manthey FA, Szelezniak EF, Nalewaja JD (1992) Phytotoxicity of bentazon with oils, surfactants and fertilizer salts. In : Adjuvants for agrochemicals (CL Foy, eds), CRC Press, Boca Raton, FL, 473-483

Matringe M, Camadro JM, Labbe P, Scalla R (1989) Protoporphyrinogen oxidase as a molecular target for diphenyl ether herbicides. Biochem J 260, 231-235 
Mine A, Matsunaka S (1975) Mode of action of bentazon: Effect on photosynthesis. Pestic Biochem Phys 5, 444-450

Morse PM (1978) Some comments on the assessment of joint action in herbicide mixtures. Weed Sci 26 , 58-71

Morvan Y, Petat JM (1992) Contrôle de la flore adventice dicotylédone en postlevée du poids protéagineux avec l'association bentazone + pendiméthaline. In : XVe Conf Columa. J Intern Lutte Mauvaises Herbes, Versailles, 795-798

Rao SR, Harger TR (1981) Mefluidide-bentazon interactions on soybeans (Glycine max) and red rice (Oryza sativa). Weed Sci 29, 208-212

Retzlaff G, Berghaus R (1988) Investigation of the antagonism of bentazone on sethoxydim activity.
In : Factors affecting herbicidal activity and selectivity, EXRS Symposium, 139-144

Taylor FE, Cobb AH, Davies LG (1980) The effect of bentazone on stomatal behavior in Chenopodium album L. New Phytol 85, 369-376

Wanamarta G, Penner D, Kells JJ (1989) The basis of bentazon antagonism on sethoxydim absorption and activity. Weed Sci 37, 400-404

Wehtje G, Wilcut JW, McGuire JA (1992) Influence of bentazon on the phytotoxicity of paraquat to peanuts (Arachis hypogaea) and associated weeds. Weed Sci 40, 90-95

Wichert RA, McCarty JT, Talbert RE (1989) Potential of a Gramoxone/Basagran tank mixture for postemergence snap bean weed control. In : Proc Annu Meet, Fayetteville 110, 130-132 